Methods This randomised controlled trial compared an asthma-tailored PR programme to usual care (UC) in participants with uncontrolled asthma and BMI $\geq 25 \mathrm{~kg} / \mathrm{m}^{2}$. PR comprised an hour of education and of exercise each week for eight weeks. Primary outcome was difference in change in Asthma Quality of Life Questionnaire (AQLQ) in PR versus UC groups post intervention. Secondary outcomes included difference in change in other asthma outcomes including asthma control questionnaire-6 (ACQ6), Medical Research Council (MRC) dyspnoea score, six-minute walk distance (6MWD) and post-exercise Borg breathlessness score. Responder analyses compared proportions reaching the minimum clinically important difference (MCID) for AQLQ and ACQ6.

Results 95 participants were randomised 1:1 to PR or UC; median age was 54, with $60 \%$ female and median BMI $33.8 \mathrm{~kg} / \mathrm{m}^{2}$. 18 participants withdrew prior to second visit, meaning 77 were included in analysis. Median (IQR) change in AQLQ was not significantly different: $0.3(-0.2$ to 0.6$)$ in $\mathrm{PR}$ and $-0.1(-0.5$ to 0.4$)$ in UC, $p=0.139$. There was no difference in proportion reaching MCID for improvement in AQLQ: $13(39 \%)$ in PR and 10(23\%) in UC, $p=0.184$. Mean change in ACQ6 was significantly different: $-0.4(95 \%$ CI -0.6 to -0.2$)$ in $\mathrm{PR}$ and $0(-0.3$ to +0.3$)$ in UC, $\mathrm{p}=0.015^{*}$. In ACQ6 responder analysis, MCID was reached by 18 participants in PR group (54.5\%) versus 10 in UC (22.7\%), $\mathrm{p}=0.009 *$. Changes in MRC dyspnoea score $\left(\mathrm{p}=0.022^{*}\right)$, 6MWD $\left(p=0.035^{*}\right)$ and Borg breathlessness $\left(p=0.015^{*}\right)$ were significantly different in favour of PR. A post-hoc analysis of PR group revealed baseline FeNO was significantly lower in ACQ6 responders (median (IQR) 18(8.5-41)) than non-responders $(47(17-71)), \mathrm{p}=0.020^{*}$; and in AQLQ responders $(14$ $(8.5-44.5))$ compared to non-responders (40(19-71)), $\mathrm{p}=0.038 \%$.

Conclusion Pulmonary rehabilitation improves asthma control and reduces perception of breathlessness in participants with difficult-to-control asthma associated with elevated BMI. It should be considered as additional therapy for this group. Lower FeNO in PR responders suggests it may be of most value in type-2 low phenotype obese asthma.

\section{S34 A MULTI-DISCIPLINARY APPROACH ENSURING SUCCESSFUL TRANSITION FROM PAEDIATRIC TO ADULT ASTHMA CARE - A FOCUS ON TREATMENT ADHERENCE}

${ }^{1} \mathrm{~N}$ Stewart-Kelcher, ${ }^{1} \mathrm{G}$ d'Ancona, ${ }^{1} \mathrm{~A}$ Hearn, ${ }^{1} \mathrm{~L}$ Thomson, ${ }^{1} \mathrm{M}$ Fernandes, ${ }^{1} \mathrm{C}$ Roxas, ${ }^{2} \mathrm{R}$ lles, ${ }^{3}$ A Gupta, ${ }^{1} J$ Dhariwal, ${ }^{4} \mathrm{DJ}$ Jackson, ${ }^{1}$ AM Nanzer. ${ }^{1}$ Guy's Severe Asthma Centre, Guy's and St Thomas' NHS Foundation Trust, London, UK; ${ }^{2}$ Paediatric Respiratory Department, Evelina Children's Hospital, London, UK; ${ }^{3}$ Paediatric Respiratory Department, Kings College Hospital, London, UK; ${ }^{4}$ Asthma UK Centre, King's College London, UK, London, UK

\subsection{6/thorax-2021-BTSabstracts.40}

Background Adolescence is a high-risk time for young asthma patients, with increased risk of asthma-related morbidity and mortality. Evidence suggests that in adolescence, over half of prescribed inhaled corticosteroid (ICS) prescriptions are not adhered to. This, a modifiable cause of troublesome symptoms, significant risk of exacerbations and death, warrants attention. Adolescents with severe asthma are seen at Guy's Young Adult Asthma Service (YAAS), having transitioned from the Children's Hospitals at King's College Hospital and the Evelina, where they are seen jointly by paediatric and adult teams during the transition period. They are supported during that time by a dedicated multi-disciplinary team including pharmacists supporting adherence.

Methods We conducted a retrospective review of patients transitioning from the paediatric teams to YAAS between October 2018 and September 2019. Patient's demographic and clinic characteristics and their adherence, quantified via Medicines Possession Ratio (MPR), the number of prescriptions issued compared with those expected to be issued, were recorded at the last joint paediatric-adult clinic appointment (transition) and again after 12 months within the adult service. Adherence was defined as poor $<50 \%$ MPR, suboptimal $<75 \%$ MPR and optimal $>75 \% \mathrm{MPR}$.

Results 25 patients (68\% female) with a mean age of 17.89 $( \pm 0.83)$ transitioned to the adult service. At transition, adherence was optimal in 10/25 (40\%), suboptimal in $3(12 \%)$ and poor in $12(48 \%)$ patients. The mean blood eosinophil count (BEC) was $0.41 \times 10^{9} / 1( \pm 0.33)$, fraction of exhaled nitric oxide (FeNO) $73.2 \mathrm{ppb}( \pm 59)$ and FEV1\% predicted $89 \%$ $( \pm 10.7)$. After 12 months, 22/25 patients remained under the

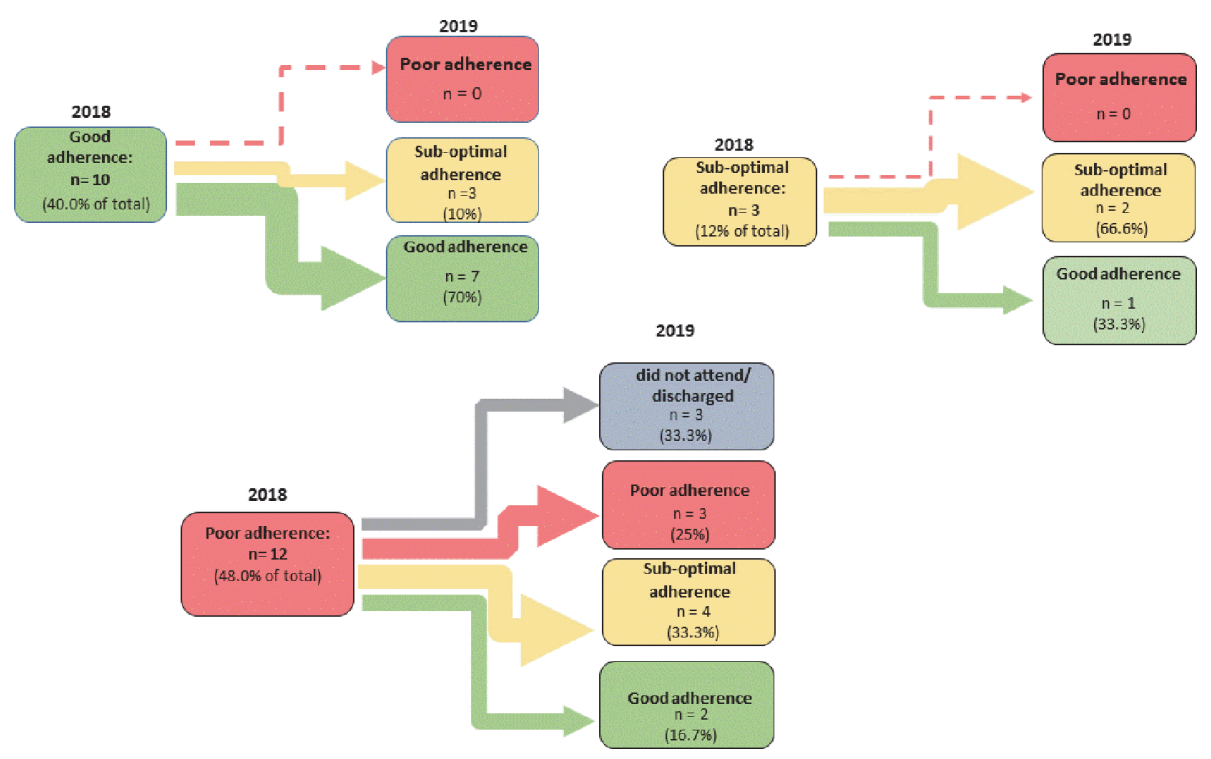

Abstract S34 Figure 1 Change in adherence patterns over 12 months 
adult service, 1 patient had been discharged and 2 patients did not attend. Adherence was optimal in 10 (44.5\%), suboptimal in $9(40.9 \%)$ and poor in $3(13.64 \%)$ patients (see figure 1). Their mean BEC was $0.35 \times 10^{9} / 1( \pm 0.33)$, FeNO 48.7 ppb $( \pm 38.1)$ and FEV1\% was $86.8 \%( \pm 12.2)$.

Conclusion While there was an incremental improvement in adherence during the first year of adult care with resultant improvements in asthma biomarkers, on-going support is needed to further increase medicines use and to ensure this behaviour persists.

\section{S35 CREATING BEHAVIOURAL PERSONAS TO DRIVE BETTER DESIGN IN HEALTH TECHNOLOGY FOR ASTHMA SELF- MANAGEMENT}

${ }^{1} \mathrm{~A}$ Fallas, ${ }^{1} \mathrm{~A}$ Cumella, ${ }^{1} \mathrm{~A}$ Whittamore, ${ }^{1} \mathrm{~B}$ West, ${ }^{1} \mathrm{C}$ Cheung, ${ }^{1} \mathrm{~J}$ Shopland, ${ }^{2} \mathrm{~A}$ Herbec, ${ }^{2} \mathrm{C}$ Stefanidou, ${ }^{2} \mathrm{~L}$ Porter, ${ }^{2} \mathrm{~N}$ Gold, ${ }^{2} \mathrm{P}$ Lacey, ${ }^{2} \mathrm{P}$ Bondaronek, ${ }^{2} \mathrm{~T}$ Papakonstantinou, ${ }^{2} \mathrm{~V}$ Mallion, 'S Walker. ${ }^{1}$ Asthma UK and British Lung Foundation, London, UK; ${ }^{2}$ PHE Behavioural Insights Team, Public Health England, London, UK

\subsection{6/thorax-2021-BTSabstracts.41}

Introduction and Objectives The health burden from asthma can be reduced through better provision of basic care and better self-management. Most health technology products tend to target a narrow range of behaviours with limited behaviour change techniques (BCTs), take a homogeneous approach towards the diverse population of people with asthma and have poor uptake.

Objective to identify and characterise distinct behavioural self-management archetypes among UK adults with asthma with the aim of creating behavioural personas that can be used by product developers to better address the needs of people with asthma.

Methods We conducted a scoping review of grey and academic literature, followed by workshops with subject matter experts to identify key behaviours and influences relevant to asthma self-management. We then conducted a rapid review and behavioural analysis on these key behaviours which were then synthesised into a behavioural systems map. A survey was constructed to explore a subset of key behaviours and influences in more detail including asthma management, asthma control, inhaler use, support seeking, monitoring, and technology use. The survey was administered to 2,324 people reflective of the UK adult asthma population. The results were analysed and synthesised using mixed methods. Data were segmented using Multiple Correspondence Analysis and k-means cluster analysis, and further statistical analysis was performed to identify factors independently associated with adherence behaviour. The results were synthesised into behavioural personas that characterise people with optimal vs. suboptimal preventer inhaler adherence in behavioural terms, alongside relevant design prompts and suggested BCTs.

Results Segmenting by inhaler use revealed behaviours distributed as shown in figure 1 . Segmenting by adherence to preventer-type inhalers alone revealed pronounced differences between optimal and sub-optimal behaviour clusters in terms of age and behavioural factors (including: skills, decision making, behavioural regulation, environmental opportunities, attitudes, motives, intentions, beliefs, identity, and emotions).

Conclusions We have developed unique insight into behaviours of people with asthma and the influences on these behaviours.

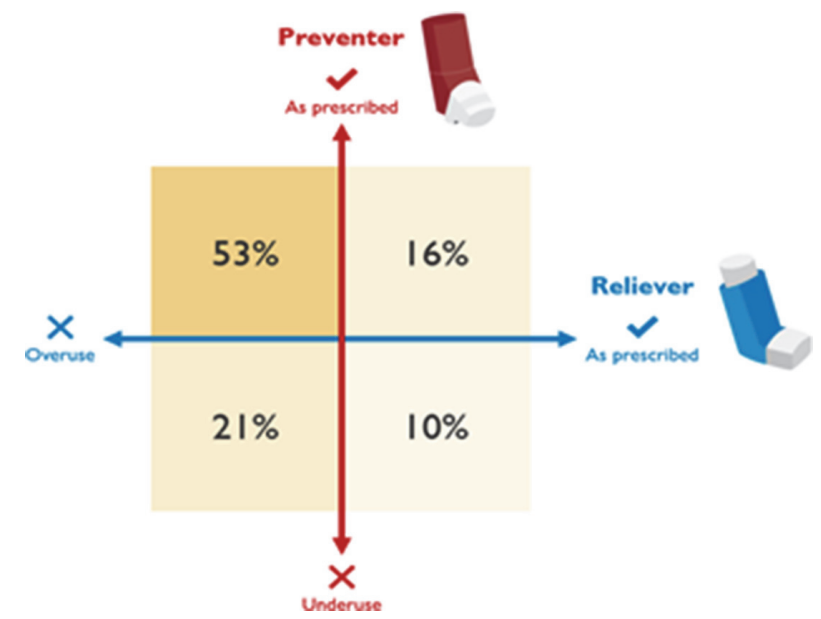

\section{Abstract S35 Figure 1}

We believe this work can contribute to a paradigm shift in the design of asthma health technology products, towards targeting new behaviours and their influences for change and ultimately driving better self-management and fewer asthma deaths.

\section{S36 PRESCRIBING PATTERNS AND TREATMENT ADHERENCE IN PATIENTS WITH ASTHMA DURING THE COVID-19 PANDEMIC}

${ }^{1} \mathrm{H}$ Dhruve, ${ }^{1} \mathrm{~J}$ Dhariwal, ${ }^{1} \mathrm{G}$ d'Ancona, ${ }^{2} \mathrm{~S}$ Holmes, ${ }^{1} \mathrm{~A}$ NanzerKelly, ${ }^{1} \mathrm{DJ}$ Jackson. ${ }^{1}$ Guys and St Thomas, London, UK; ${ }^{2}$ Park Medical Practice, Somerset, UK

\subsection{6/thorax-2021-BTSabstracts.42}

Introduction and Objective The COVID-19 pandemic has witnessed a reduction in asthma exacerbations in the UK. Several factors may underpin this, including reduced transmission of seasonal viruses and improved use of or adherence to inhaled corticosteroids (ICS). This study aims to investigate whether ICS use has changed during the pandemic for patients with asthma.

Methods Using the OpenPrescribing database, we analysed prescribing patterns of ICS, salbutamol and peak flow meters from January 2019 to January 2021 across England. Additionally, using a sample asthma cohort from 3 primary care practices, we assessed individual prescription patterns and ICS adherence across the two-year period. ICS adherence has been defined according to the medication possession (MPR) ratio: good $(\geq 75 \%)$, sub-optimal $(50-74 \%)$, poor $(25-49 \%)$ and non-adherence $(<25 \%)$.

Results A sharp increase in national ICS prescriptions was observed at the start of the pandemic in March 2020 representing a 50\% increase compared to February 2020. Thereafter national ICS prescription rates appear to have returned to normal levels. The sample asthma cohort included 1132 patients (762 patients treated with ICS across 2019 and 2020). Overall, adherence to ICS improved in 2020 $(P<0.001)$, with the proportion of patients meeting 'good adherence' $(\geq 75 \%)$ increasing from $34 \%$ to $42 \%(P<0.001)$. Analysis of this cohort suggested the March 2020 spike predominantly reflected improved adherence rather than a hoarding effect of multiple inhalers or new prescriptions for ICSnaïve individuals. Increasing age was associated with higher 